\title{
Nanoscale
}

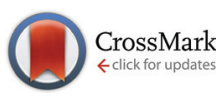

Cite this: Nanoscale, 2016, 8, 17963

\section{Optimizing CuO p-type dye-sensitized solar cells by using a comprehensive electrochemical impedance spectroscopic study $\dagger$}

\author{
Oliver Langmar, ${ }^{a}$ Carolina R. Ganivet, ${ }^{\mathrm{b}}$ Gema de la Torre, ${ }^{\mathrm{b}}$ Tomás Torres,,${ }^{\mathrm{b}, \mathrm{c}}$ \\ Rubén D. Costa*a and Dirk M. Guldi*a
}

\begin{abstract}
We introduce a novel and comprehensive approach for the evaluation and interpretation of electrochemical impedance spectroscopy (EIS) measurements in p-type DSSCs. In detail, we correlate both the device performance and EIS figures-of-merit of a series of devices in which, the calcination temperature, film thickness, and electrolyte concentration have been systematically modified. This new approach enables the separation of the different processes across the dye/semiconductor/electrolyte interface, namely the unfavorable charge recombination and the favorable electron injection/regeneration processes. In addition, studies on non-sensitized $\mathrm{CuO}$ and $\mathrm{NiO}$ electrodes provide insights into their affinity towards a reaction with the electrolyte - $\mathrm{CuO}$ is far less reactive towards the polyiodide species. Overall, this work underlines the superior features of $\mathrm{CuO}$ with respect to $\mathrm{NiO}$ for $\mathrm{p}$-DSSCs and demonstrates a comprehensive optimization of the CuO-based DSSCs with respect to the device architecture by the aid of EIS analysis.
\end{abstract}

Received 12th July 2016

Accepted 29th September 2016

DOI: $10.1039 / \mathrm{c} 6$ nr05507a

www.rsc.org/nanoscale

The aforementioned renders copper delafossites valuable competitors to the already established NiO-DSSCs. ${ }^{7}$

We have recently probed the suitability of CuO-DSSCs, achieving efficiencies of $0.10 \%$ and $0.19 \%$, based on an iodide- or cobalt-electrolyte, respectively. ${ }^{8}$ In this work, a study about the detailed optimization of CuO-DSSCs is presented by combining device analysis with a comprehensive electrochemical impedance spectroscopic (EIS) study. We evaluate the impact of the $\mathrm{CuO}$ electrode fabrication - calcination temperature and electrode thickness - and the $\mathrm{I}^{-} / \mathrm{I}_{2}$ electrolyte ratio on the device performance. Please notice that although the cobalt-based redox couple yields higher efficiencies for CuO-DSSCs, the device stability is compromised due to the rapid crystallization of this electrolyte onto the electrode surface. Therefore, a meaningful EIS characterization is not possible. To this end, our work provides a guideline for the development of photocathodes for DSSCs.

EIS characterization of n-type DSSCs is a well-established technique, ${ }^{9-14}$ but detailed EIS investigations on p-type DSSCs are surprisingly scarce. Wu et al. were among the first to apply EIS to p-type DSSCs and to interpret the obtained parameters. ${ }^{15}$ The recombination resistance $\left(R_{\text {rec }}\right)$ was probed at different applied voltages, showing a unique behavior compared to $\mathrm{TiO}_{2}$ based DSSCs. This fundamental difference was attributed to recombination between the excited state of the reduced photosensitizer and the NiO semiconductor. ${ }^{16} \mathrm{~A}$ comparable study for other p-type DSSC electrodes including $\mathrm{CuO}$

\footnotetext{
${ }^{a}$ Department of Chemistry and Pharmacy, Interdisciplinary Center for Molecular Materials, University of Erlangen-Nürnberg, Egerlandstr. 3, 91058 Erlangen, Germany.E-mail: ruben.costa@fau.de,dirk.guldi@fau.de

Sciences (IAdChem), C/Francisco Tomás y Valiente 7, 28049 Madrid, Spain

'Instituto Madrileño de Estudios Avanzados (IMDEA)-Nanociencia c/ Faraday, 9, Cantoblanco, 28049 Madrid, Spain

$\dagger$ Electronic supplementary information (ESI) available. See DOI: 10.1039/ c6nr05507a
} 
is still missing. Thus, we believe that our work may assist in understanding the full potential of $\mathrm{CuO}$ electrodes in p-type DSSCs. ${ }^{17,18}$ Firstly, a new and comprehensive approach for the interpretation of EIS measurements, which was validated by a thorough comparison with different devices, is described. This is compared to already known models for EIS characterization of p-type DSSCs. Secondly, a comparison between $\mathrm{NiO}$ and $\mathrm{CuO}$ based photocathodes is performed to probe the inherent differences between both materials. Thirdly, device parameters for p-type DSSCs, such as calcination temperature, electrode thickness, and electrolyte composition are optimized, by performing a detailed and comprehensive EIS study.

\section{Experimental details and characterization}

$\mathrm{CuO}$ pastes of $15 \mathrm{wt} \%$ were prepared by diluting ethylcellulose (Sigma-Aldrich), CuO powder (CAS no. 1317-38-0, Iolitec) and triacetin (Sigma-Aldrich) in ethanol. The suspension was stirred for several days before being used for doctor blading. Prior to doctor blading, fluorine doped tin oxide glass slides (FTO, $8 \Omega$ per square, Pilkington, XOP Glass) were washed in an ultrasonic bath in solutions of acetone, tenside solution, water and isopropanol for 15 minutes each. Afterwards, the slides were cleaned in an ozone lamp cleaner (Jelight company, Inc.). The pastes were applied by doctor blading onto the FTO substrates, with the help of a Scotch tape mask (total area of the electrodes was $0.2 \mathrm{~cm}^{2}$ ). In the next step, the prepared films were calcinated at $300{ }^{\circ} \mathrm{C}$ on a hotplate for 30 minutes and then immersed into the $\mathrm{Zn}(\mathrm{II})$ phthalocyanine (ZnPc2) dye solution (see Fig. S2 $\dagger)\left(c=1 \times 10^{-4} \mathrm{M}\right.$ in ethanol) for 1 hour. The thickness of the electrodes was controlled by either increasing the amount of ethanol in the paste $(\approx 30 \%$ more ethanol) or by using up to three layers of scotch tape (thickness of one layer of scotch tape is $55 \mu \mathrm{m}$ ) during doctor blading and was measured with a profilometer (Bruker OM DektakXT).

The final devices were assembled by pressing the $\mathrm{CuO}$ electrodes and the $\mathrm{Pt}$ counter electrode $\left(5 \mathrm{mM} \mathrm{H}_{2} \mathrm{PtCl}_{6}\right.$ on FTO baked at $400{ }^{\circ} \mathrm{C}$ ) with a Syrlin (Solaronix, $25 \mu \mathrm{m}$ ) at $150{ }^{\circ} \mathrm{C}$. The electrolyte consists of $1 \mathrm{M}$ LiI (Sigma-Aldrich) and $0.2 \mathrm{M} \mathrm{I}_{2}$ (Sigma-Aldrich) (if not stated otherwise) in acetonitrile. The electrolyte was filled into the cell through two pre-drilled holes in the counter electrode and the holes were afterwards sealed with Syrlin foil and an additional glass slide. Five devices have been measured each to ensure the reproducibility of our results.

The assembled devices were tested under dark and 1 sun AM 1.5 standard illumination conditions. To avoid the influence of scattered light a mask with a total surface area of $0.28 \mathrm{~cm}^{2}$ was used. Photocurrent curves were measured with a potentiostat (Metrohm PGSTAT30) in the range of -0.2 to 0.05 $\mathrm{V}$, with the counter electrode connected to the $\mathrm{CuO}$ and the working electrode connected to the platinum electrode. Electrochemical impedance measurements were measured with the same potentiostat under dark and AM 1.5 illumination conditions in the frequency range of $0.01 \mathrm{~Hz}$ to $100 \mathrm{kHz}$. The AC amplitude has been set to $10 \mathrm{mV}$ to ensure a linear response of the investigated devices. Multiple impedance measurements with different applied bias have been performed for each device starting at the $V_{\text {oc }}$ going in $10 \mathrm{mV}$ steps until the short-circuit current $\left(J_{\mathrm{sc}}\right)$ conditions have been reached. After each impedance measurement, a currentvoltage curve was recorded to document the constant of the device figures-of-merit. This corroborates the stability of the devices under investigation. Furthermore, the Kronig-Kramers (KK) test confirmed that the performed measurements fulfilled the standard EIS criteria of causality, linearity, stability, and finiteness - please see the ESI Fig. S1 and Table $\mathrm{S} 1 \dagger$ for an exemplary KK-Test and the respective modeling errors. Please note, that the KK-Test has been carried out as a complex fit using NOVA 1.11 software. $^{19}$ Furthermore, the obtained Nyquist plots were also fitted using the NOVA 1.11 software, where an appropriate model (vide infra) and a weighting factor of $1 /|Z|^{2}$ was used for the fittings to obtain the impedance parameters - Table $\mathrm{S} 2 \dagger$ for exemplary fitting values. The iRdrop has been taken into account by correction via eqn (1): ${ }^{10,16}$

$$
V_{\text {app }}=V_{\mathrm{f}}-J R_{\mathrm{s}}
$$

with $V_{\text {app }}$ being the applied voltage, $V_{\mathrm{f}}$ the potential related to the separation of Fermi levels, $J$ the current density at $V_{\mathrm{f}}$, and $R_{\mathrm{S}}$ as the sum of $r_{\mathrm{S}}$ and $R_{\mathrm{CE}}$ extracted from the EIS measurements. Incident photon-to-current efficiencies (IPCE) have been measured in the spectral range of 320 to $800 \mathrm{~nm}$ by illuminating the p-type CuO DSSCs with a xenon arc lamp over a Cornerstone $260 \frac{1}{4} \mathrm{~m}$ Monochromator equipped with a Merlin digital radiometric lock-in-system. X-ray diffraction (XRD) assays were performed using a D8Advance (Bruker AXS) equipped with a $\mathrm{Cu} \mathrm{K} \alpha$ radiation source. Scanning electron microscopy (SEM) measurements were performed using a Zeiss Gemini 55 Ultra electron microscope. Raman spectroscopy assays were performed with a Reflex Confocal Raman microscope (Renishaw) with an excitation wavelength of $532 \mathrm{~nm}$.

\section{Results and discussion}

\section{Description of the EIS model for p-type DSSCs}

First, a model for the data interpretation needs to be established. Fig. S2† displays the Nyquist plots of CuO-DSSCs under 1 sun illumination and in the dark. On one hand, the arc in the high frequency region relates to reactions across the platinum counter electrode/electrolyte interface. On the other hand, the semicircle in the low frequency region corresponds to the electron injection, regeneration, and recombination processes across the dye/semiconductor/electrolyte interface depending on the applied voltage and illumination conditions. $^{15}$

The processes at the different interfaces are correlated with the elements in the electrical circuit model Fig. S2. $\dagger$ In particu- 
lar, $R_{\mathrm{S}}$ describes the series resistance and includes contributions stemming from the FTO sheet resistance, the resistance of the cables, and the resistance of the clamps or pins used for contacting. ${ }^{15} R_{\mathrm{Pt}}$ is associated with the charge transfer (CT) process across the counter electrode interface and is connected in parallel with the constant phase element (CPE) with $C_{\mathrm{Pt}}$ as the Helmholtz capacitance. In the case of DSSCs, CPE is typically expressed as capacitance via eqn (2): ${ }^{20}$

$$
C=\frac{\left(Y_{0} R\right)^{1 / \alpha}}{R}
$$

in which $Y_{0}$ is a constant $\left[\mathrm{Ss}^{\alpha}\right], \alpha$ an empirical constant, and $R[\Omega]$ the parallel connected resistance. The second resistanceCPE element consists of either $R_{\text {rec }}$ or $R_{\mathrm{CT}} \cdot R_{\text {rec }}$ is associated with the recombination resistance of holes in the semiconductor with the electrolyte under dark conditions, while $R_{\mathrm{CT}}$ relates to the $\mathrm{CT}$ resistance of different processes under 1 sun illumination - vide infra. $C_{\mu}$ is the chemical capacitance of the dye/semiconductor/electrolyte interface and is often compared to the density of states (DOS). ${ }^{14}$ For an ideal semiconductor, $C_{\mu}$ is supposed to increase exponentially with the applied potential, since the Fermi level $\left(E_{\mathrm{F}}\right)$ converges either with the conduction band (CB) of a n-type or with the valence band (VB) of a p-type semiconductor. In n-type $\mathrm{TiO}_{2^{-}}$and ZnO-DSSCs this behavior has widely been noted, ${ }^{21}$ but never for p-type NiO-DSSCs. ${ }^{22}$ It should be noted that a number of different models, which include additional parameters, such as transmission line and Warburg diffusion elements, have frequently been used for EIS measurements of DSSCs. ${ }^{23,24}$ In the Nyquist plots of our EIS assays, we have not observed any characteristic transmission line features. Quite likely this is due to the very small transport resistance $\left(R_{\mathrm{tr}}\right)$. Furthermore, the Warburg diffusion feature of the electrolyte, which is sometimes noted in n-type DSSCs, was completely absent in our measurements. Thus, we used the aforementioned simplified model, which has been successfully used in the past for the characterization of $n$ - and p-type DSSCs. ${ }^{15,25}$

Next, the time constant and/or hole lifetime is determined, using eqn (3), in which $R$ and $C_{\mu}$ are taken from the fittings, or using its effective rate constant $k_{\text {eff }}$ as shown in eqn (4). The latter is obtained from the low frequency arc in the Nyquist plot - Fig. S2:† 13,26

$$
\begin{gathered}
\tau_{\mathrm{n}}=R \times C_{\mu} \\
\tau_{0}=\frac{1}{k_{\text {eff }}}=\frac{1}{\omega}=\frac{1}{2 \pi f}
\end{gathered}
$$

with $\omega$ the angular frequency $\left[1 \mathrm{~s}^{-1}\right]$ and $f$ the frequency $[\mathrm{Hz}]$ of the corresponding Nyquist maximum.

For the analysis and fitting of EIS data a myriad of software and tools exists, and each one of them displays the Nyquist plot frequency in a different way, that is, as $\omega$ or $f$. Thus, care has to be taken in which way the obtained frequency is inserted into eqn (4) to ensure a correct calculation of the lifetime to avoid misunderstandings when the lifetimes are compared to literature values. In n-type DSSCs, the two lifetimes have different origins as explained by Bisquert et $a l^{13}$ Lifetimes obtained from $k_{\text {eff }}$, are referred to as free electron lifetimes $\tau_{0}$ as they transfer through the CB. Whereas the apparent lifetimes $\tau_{\mathrm{n}}$ - eqn (3) - are affected by the constant trapping or detrapping of electrons in bandgap states. Notable, $\tau_{\mathrm{n}}$ is slightly longer than $\tau_{0}$ for $\mathrm{TiO}_{2}$ based devices.

Finally, the effective diffusion lengths $L_{\text {eff }}$ - eqn (5) - the effective diffusion coefficients of the charge carriers $D_{\text {eff }}-$ eqn (6) - and the charge collection efficiencies $\eta_{\text {cc }}$ can be calculated - eqn (7): ${ }^{16,26,27}$

$$
\begin{gathered}
L_{\text {eff }}=d \times \sqrt{\frac{R_{\text {rec }}}{R_{\mathrm{CT}}}}=\sqrt{D_{\text {eff }} \times \tau_{0}} \\
D_{\text {eff }}=\frac{R_{\text {rec }}}{R_{\mathrm{CT}}} \times d^{2} \times k_{\text {eff }} \\
\eta_{\mathrm{cc}}=1-\frac{R_{\mathrm{CT}}}{R_{\text {rec }}}
\end{gathered}
$$

$d$ is the thickness of the electrode. Basically, $L_{\text {eff }}$ describes the distance a hole travels throughout the VB before recombination with an electron. The diffusion of holes throughout VB is described by $D_{\text {eff }}$ and it is closely related to $L_{\text {eff }}$, namely the $R_{\text {rec }}$ to $R_{\mathrm{CT}}$ ratio - eqn (6). The effective yields of holes at the back contact of the electrodes are usually calculated by $\eta_{\mathrm{cc}}$. It is a useful parameter in the context of comparing devices with different charge injection and recombination rates. ${ }^{8}$ It is not only enough to probe the different resistances at $V_{\text {oc }}$ conditions, but to make a complete scan over the entire applied voltage going from $V_{\text {oc }}$ to $J_{\text {sc }}$ conditions - Fig. 1. This approach is today's standard EIS procedure for characterizing n-type DSSCs. ${ }^{10,28}$ For p-type DSSCs, it was first applied by Wu et al., but only few follow-up studies have been performed. ${ }^{15,29}$ As aforementioned, the Nyquist plots serve as a basis for extrapolating $R_{\mathrm{rec}}$ and $R_{\mathrm{CT}}$, but their relation to a given process depends on the exact conditions - under illumination with 1 sun AM 1.5 conditions (light) or under no illumination (dark). In the dark, the dye is inactive and only the reaction between the electrolyte and the electrode results in dark current generation. This is a major recombination pathway in DSSCs and it is denoted as $R_{\text {rec }}$. A low $R_{\text {rec }}$ relates to a high dark current and, in turn, to low device efficiency. In other words, $R_{\text {rec }}$ should be as high as possible. By applying a voltage equal to the device $V_{\mathrm{oc}}$, the $E_{\mathrm{F}}$ of $\mathrm{CuO}$ separates from the potential of the redox couple and recombination processes become thermodynamically more favorable, resulting in low $R_{\mathrm{rec}} \mathrm{S}-$ Fig. 1. At $J_{\mathrm{sc}}, E_{\mathrm{F}}$ of $\mathrm{CuO}$ equilibrates with the potential of the electrolyte and the driving force for recombination processes turns out to be negligible. Thus, a decrease in voltage is related to an exponential increase of $R_{\mathrm{rec}}$ - eqn (8): ${ }^{12,16}$

$$
R_{\text {rec }}=R_{0} \exp \left(-\frac{q \beta}{k_{\mathrm{B}} T} V\right)
$$

with $k_{\mathrm{B}}$ being the Boltzmann's constant, $T$ the absolute temperature, $q$ the elementary charge, and $\beta$ the transfer coefficient. Typical $\beta$ values for n-type DSSCs are in the range from 0.4 to 

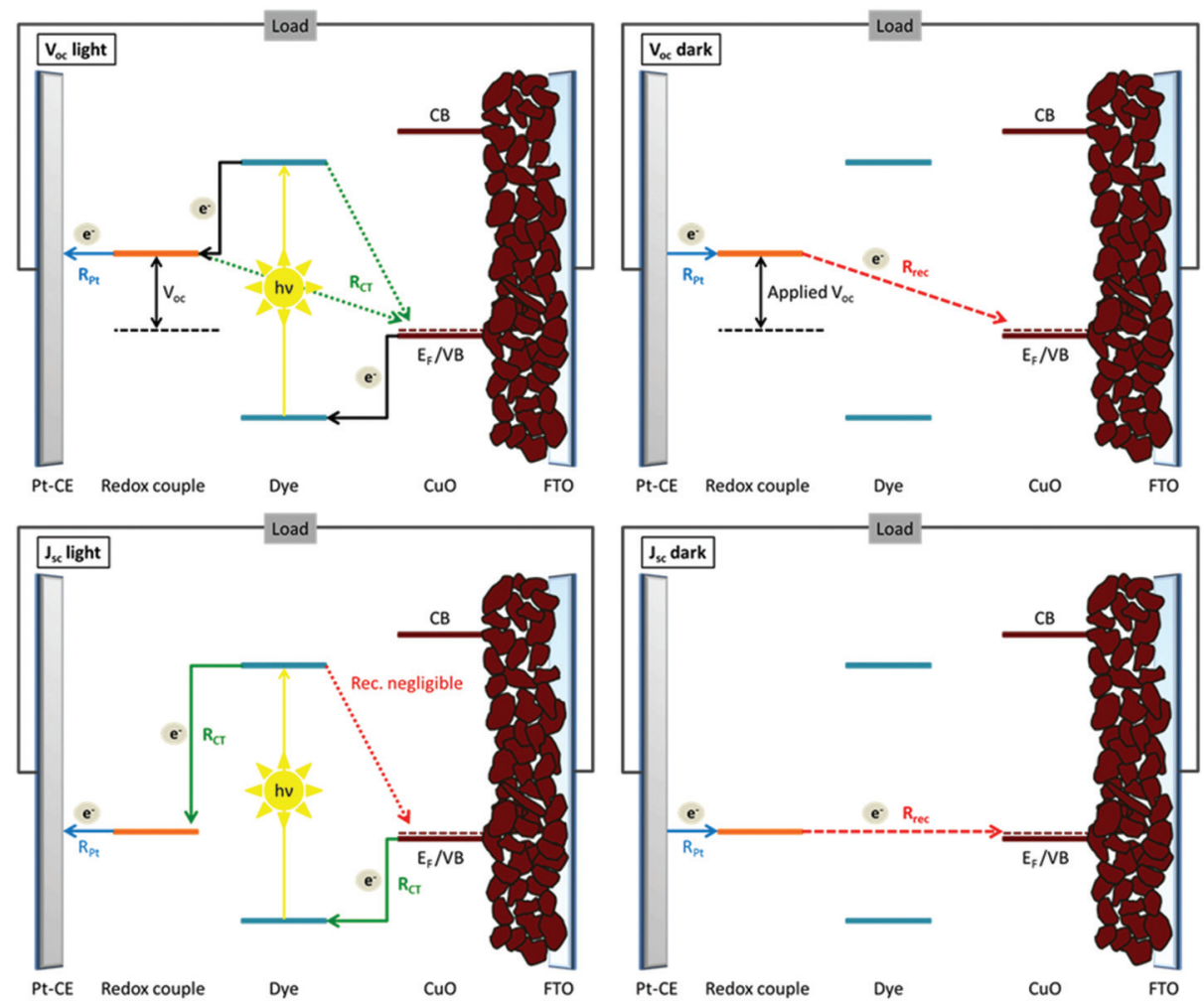

Load

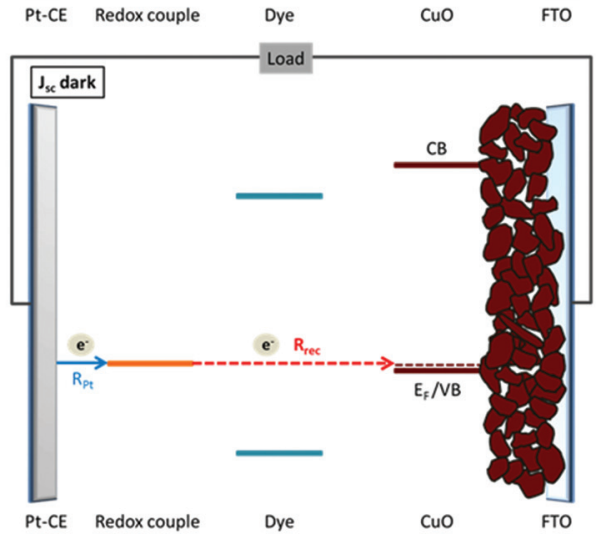

Fig. 1 Energetic diagrams of the four most characteristic conditions, under which EIS can be performed, that is, under $V_{\text {oc }}$ light (top left), $V_{\text {oc }}$ dark (top right), $J_{s c}$ light (bottom left), and $J_{\text {sc }}$ dark (bottom right). The dominant processes, to which the resistances are ascribed are shown in each diagram, that is, $R_{\mathrm{CT}}$ (green), $R_{\mathrm{rec}}(\mathrm{red})$, and $R_{\mathrm{Pt}}$ (blue). Favorable processes, like injection/regeneration, and unfavorable, like recombination, are depicted in solid and dashed arrows, respectively.

0.8. ${ }^{10,13}$ By fitting $R_{\text {rec}}$, which is presented in Fig. 2, we obtained a $\beta$ value of 1.26 , which is lower than the values obtained for NiO-based p-type DSSCs. ${ }^{15}$ A smaller $\beta$ value prompts to fewer recombinations in CuO-based DSSCs compared to devices consisting of NiO-based photocathodes. This trend has been concluded from our EIS measurements of nonsensitized $\mathrm{CuO}$-and NiO-based electrodes - vide infra.

A different scenario is noted under light, as the dye is excited. At $V_{\mathrm{oc}}$, four processes across the dye/semiconductor/ electrolyte interface determine the overall current generation. On one hand, electron injection from $E_{\mathrm{F}}$ of $\mathrm{CuO}$ to the highestoccupied-molecular-orbital (HOMO) of the excited dye and its regeneration by the electrolyte are responsible for the photocurrent. On the other hand, recombination of electrons from either the redox couple or the reduced state of the photoexcited sensitizer back to the electrode produces a recombination current - Fig. 1. At $V_{\text {oc }}$, no net current is flowing, since the recombination dominates the device and the corresponding $R_{\mathrm{CT}}$. At $J_{\mathrm{sc}}$, the photocurrent reaches its maximum and, thus, recombination its minimum. Thus, under forward conditions back electron transfer from the excited state of the reduced sensitizer to the semiconductor is negligible in EIS measurements under $J_{\mathrm{sc}}$ conditions. This is also reflected in the $\eta_{\mathrm{cc}} \mathrm{S}$ at the aforementioned conditions which are close to unity - vide infra. This leaves electron injection and regeneration of the sensitizer as the dominant processes $-R_{\mathrm{CT}}$. Both are photocurrent generating processes, which means a lower $R_{\mathrm{CT}}$ is favorable.

As shown in Fig. 2, $R_{\mathrm{CT}}$ features a linear increase in the high voltage region before it reaches a plateau at low voltage and $J_{\text {sc }}$, as the photocathode does not show insulating features as it has been observed in n-type DSSCs before. ${ }^{8,10,16}$ Furthermore, $R_{\mathrm{CT}}$ does not follow the exponential dependence described in eqn (8).

In general, NiO reveals a higher reactivity towards electrolytes throughout the voltage region than bare FTO. ${ }^{16}$ As such, a negligible leaking current across the FTO/electrolyte interface eliminates the needs for buffer layers in p-type NiO DSSCs. ${ }^{16}$ In the next section, we probe $\mathrm{CuO}$ films in a direct comparison with state-of-the-art NiO electrodes and bare FTO references. ${ }^{30,44}$ To this end, we assembled sandwich devices, consisting of bare $\mathrm{CuO}$ and NiO electrodes deposited on FTO facing a standard Pt coated FTO electrode. A mixture of $1 \mathrm{M} \mathrm{LiI/0.2} \mathrm{M} \mathrm{I}_{2}$ in a $5: 1$ ratio in acetonitrile was used as electrolyte and the corresponding $R_{\mathrm{rec}} \mathrm{S}$ and $C_{\mu} \mathrm{S}$ are summarized in Fig. 3.

\section{Comparison of bare $\mathrm{NiO}$ vs. $\mathrm{CuO}$ electrodes}

We opted for non-sensitized electrodes of comparable thickness $(1.1 \mu \mathrm{m})$ and dark conditions to exclude effects from 

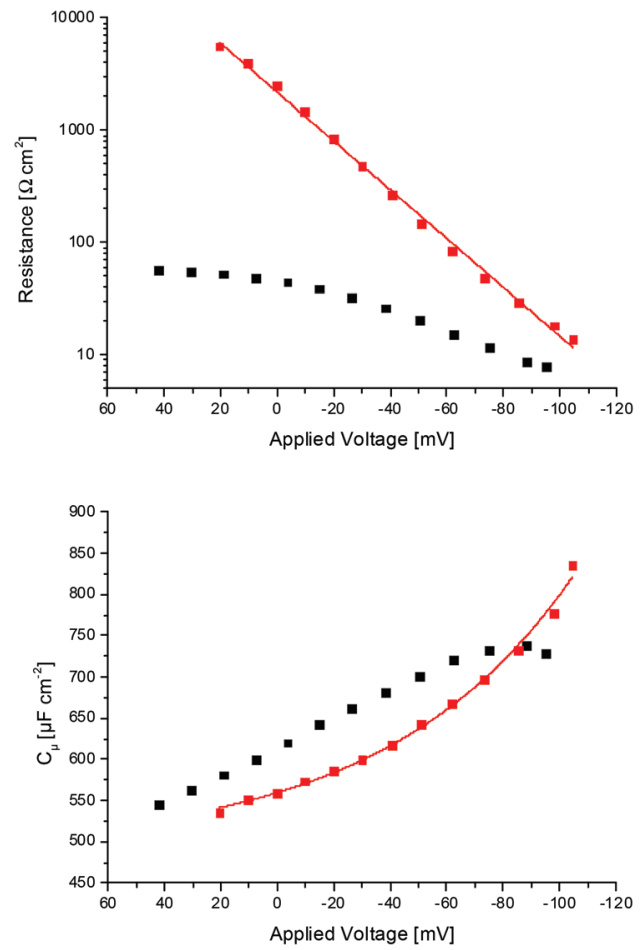

Fig. 2 Top part: $R_{\mathrm{CT}}$ (black) and $R_{\text {rec }}$ (red). Bottom part: $C_{\mu}$ under light (black) and dark (red) conditions for a p-type CuO DSSC probed over the whole voltage region. The corresponding fits according to eqn (8) $\left(R_{\text {rec }}\right)$ and $(9)\left(C_{\mu}\right)$ are shown as lines.
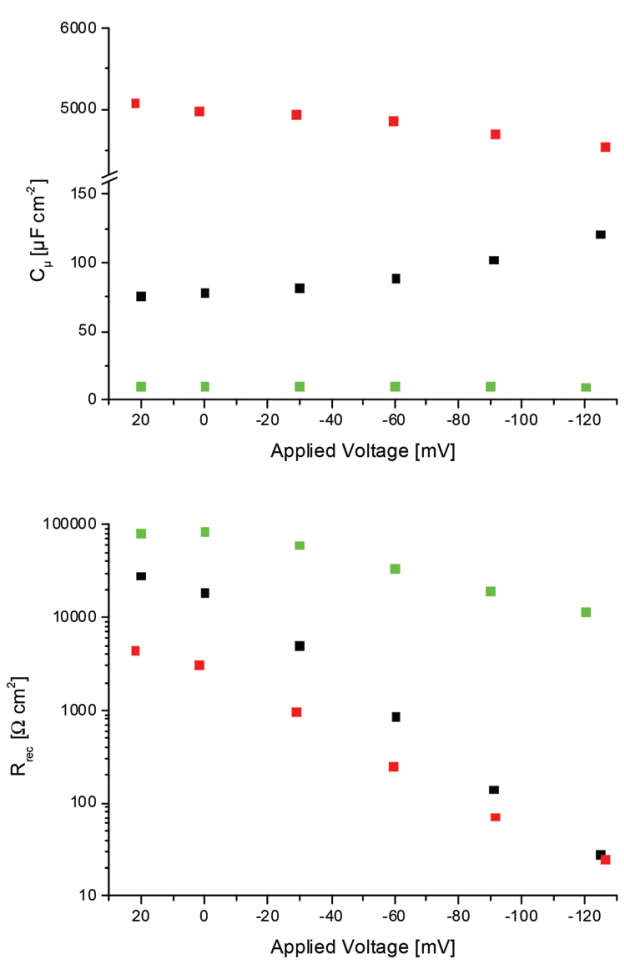

Fig. $3 R_{\text {rec }}$ (top) and $C_{\mu}$ (bottom) vs. applied voltage measured under dark for $\mathrm{CuO}$ (black), $\mathrm{NiO}$ (red) electrodes, and bare FTO (green). photoexcitation of the electrolyte or the electrode and compared them with bare FTO.

Regarding $R_{\mathrm{rec}} \mathrm{s}$, bare FTO shows the highest resistance with 11.6 and $84.8 \mathrm{k} \Omega \mathrm{cm}^{2}$ at 120 and $0 \mathrm{mV}$, respectively. In contrast, $\mathrm{CuO}$ and $\mathrm{NiO}$ electrodes exhibit only small differences in $R_{\text {rec }}-27.9 \Omega \mathrm{cm}^{2}(\mathrm{CuO})$ and $25.1 \Omega \mathrm{cm}^{2}(\mathrm{NiO})$ at $120 \mathrm{mV}$. By lowering the applied voltage, $R_{\text {rec }}$ increases and the overall differences between both electrodes is more pronounced. At zero applied bias, the resistance of $18.5 \mathrm{k} \Omega$ $\mathrm{cm}^{2}$ for $\mathrm{CuO}$ is higher compared to only $3.1 \mathrm{k} \Omega \mathrm{cm}^{2}$ for $\mathrm{NiO}$, which points to a six-times lower reactivity of $\mathrm{CuO}$ electrodes towards the redox couple when compared to NiO. Still, it is, arguable if a buffer layer might improve the device performance, since the $\mathrm{CuO}$ electrodes still show a higher reactivity towards the redox couple compared to bare FTO. The same trends emerge in the $C_{\mu}$ measurements with 9.9, 121.2, and $4546.0 \mu \mathrm{F} \mathrm{cm}$ at an applied voltage of $120 \mathrm{mV}$ for FTO, CuO, and $\mathrm{NiO}$, respectively. Higher $C_{\mu} \mathrm{s}$ suggest the presence of more charge carriers across the electrolyte/electrode interface, pointing to a higher reactivity with the electrolyte. More interesting, $\mathrm{CuO}$ electrodes feature an exponential increase in $C_{\mu}$ upon increasing voltage. This is a typical chemical capacitance behavior as expected for an ideal semiconductor. ${ }^{14}$ In stark contrast, $\mathrm{NiO}$ electrodes exhibit an almost linear behavior with a slight decrease towards higher applied voltages.

In line with the recent literature, we hypothesize that the film capacitance of $\mathrm{NiO}$ is too high and, thus, a meaningful determination of $C_{\mu}$ for NiO electrodes by means of EIS is difficult. ${ }^{22}$ In other words, calculations regarding $\tau_{\mathrm{n}}$ - according to eqn (3) - lead to large error margins with respect to $C_{\mu}$. Thus, it needs to be further discussed if EIS measurements of NiO-DSSCs can be interpreted with the standard model. Helpful in this regard would be to find alternative ways to determine $C_{\mu}$ and compare them with the obtained values of the EIS assays.

Next, p-type CuO DSSCs were sensitized with ZnPc2 and filled with the iodide-based electrolyte as previously reported. ${ }^{8}$ With a $V_{\text {oc }}$ of $102 \mathrm{mV}$ and a $J_{\text {sc }}$ of $2.78 \mathrm{~mA} \mathrm{~cm}^{-2}$ a reasonable efficiency of $0.1 \%$ was obtained - the current density $v s$. applied potential $(J-V)$ curve and Incident photon-to-current efficiency (IPCE) spectra are shown in Fig. S3.†

Since the voltage-dependent behavior of $R_{\mathrm{CT}}$ and $R_{\text {rec }}$ has already been explained - vide supra - the next important parameter to consider is the chemical capacitance $C_{\mu}$ under light and dark - Fig. 2 . In both cases, $C_{\mu}$ increases with increasing voltage due to the fact that the energy of the quasi Fermi level approaches that of the valence band (VB). Such a trend is typical for an ideal semiconductor and corroborates the relationship between measured capacitance and DOS of the mesoporous film. ${ }^{21,22}$ If $C_{\mu}$ follows an exponential law, which is the case for our measurements in the dark, it can be fit with eqn (9): $:^{21,31}$

$$
C_{\mu}=C_{0} \exp \left(\frac{\alpha}{k_{\mathrm{B}} T} V\right)
$$


With $k_{\mathrm{B}}$ as the Boltzmann's constant, $T$ the absolute temperature, and $\alpha$ as a dimensionless parameter that relates to the mean energy of the exponential distribution of localized band gap states in metal oxide semiconductors. A value of 0.39 for $\alpha$ has been obtained by fitting $C_{\mu}$ from the dark measurement, which is in the range of $\mathrm{TiO}_{2}$-based DSSCs $(\alpha=0.35-0.5) .{ }^{10,21}$

As aforementioned, there are several ways to derive the time constant for a specific process in EIS measurements. For instance, the frequency at the maximum of the bode phase plot, instead of the Nyquist plot maximum frequency was taken and used in eqn (4). ${ }^{24,27}$ This leads, however, to different values. Therefore, we calculated the time constant in accordance with eqn (3) as $\tau_{\mathrm{n}}$, with eqn (4)/the Nyquist plot maximum frequency as $\tau_{\mathrm{o}}$ (inverse of $k_{\text {eff }}$ ), and eqn (4)/the frequency of the maximum in the bode phase plot as $\tau_{\mathrm{o}}-$ Fig. S4.† Overall, the time constants increase as the voltages are decreased before reaching a plateau around $J_{\mathrm{sc}}$ condition. For $\tau_{\mathrm{n}}$ - eqn (3) - this is not surprising, since the product of $R_{\mathrm{CT}}$ and $C_{\mu}$ is governed by the resistance and, thus, the trends regarding $R_{\mathrm{CT}}$ and $\tau_{\text {light }}$ should be comparable. For $\tau_{\mathrm{o}}$ - eqn (4)/the Nyquist plot maximum frequency - a similar trend was determined. Please note, that $\tau_{\mathrm{n}}$ and $\tau_{\mathrm{o}}$ yield the exact same values of the lifetime across the whole voltage range. If, the frequency of the maximum in the bode phase plot is used, the differences are as large as $70 \%$. Some groups have proposed for n-type DSSCs that the maximum of the bode phase plot refers to the electron transport in the semiconductor electrode, ${ }^{32}$ but further investigations are needed for p-type DSSCs if this assumption also holds true. This was, however, not the scope of our current investigations. Thus, we propose that the determination of time constants should be done by eqn (3), since it takes the effect of traps into account and is the most accurate method.

Finally, we denoted the time constants for light and dark as $\tau_{\text {light }}$ and $\tau_{\text {dark }}$, respectively - Fig. S4. $\dagger$ In CuO-DSSCs, $\tau_{\text {light }}$ and $\tau_{\text {dark }}$ followed the trend of its related resistances as expected. Although $\tau_{\text {dark }}$ is several orders of magnitude larger than $\tau_{\text {light }}$, care has to be taken to which conditions these values refer to. $\tau_{\text {dark }}$ and $\tau_{\text {light }}$ at $V_{\text {oc }}$ relate to recombination processes, that is, the hole lifetime. $\tau_{\text {dark }}$ should be larger at $V_{\text {oc }}$, since only recombination between the electrolyte and the semiconductor is present, whereas for $\tau_{\text {light }}$ additional recombination between the reduced state of the photoexcited sensitizer and injected holes should be considered.

Upon decreasing the applied voltage in the dark, the quasi Fermi level is moving away from the VB edge, lowering the density of accessible states in the $\mathrm{CuO}$ electrode. Fewer charge carriers are present for recombination and $\tau_{\text {dark }}$ increases accordingly. The same trend is observed for $\tau_{\text {light }}$ until reaching $J_{\mathrm{sc}}$ conditions, where it starts to plateau. The relation of $\tau_{\text {light }}$ at $J_{\mathrm{sc}}$ either to the regeneration or to the injection is less than straightforward and depends on calcination temperature, electrode thickness, electrolyte composition, etc. - vide infra.

Finally, $\eta_{\mathrm{cc}}, L_{\mathrm{eff}}$, and $D_{\text {eff }}$ all represent the ratio between electron injection/regeneration and recombination processes Fig. S4. $\dagger$ Throughout the complete voltage range, we note changes for all of them in the form of increases with a decrease in voltage. This is reasonable, owing to the fact at $V_{\mathrm{oc}}$ recombination takes over and most of the injected charge carriers recombine, resulting in lower $\eta_{\mathrm{cc}}$, shorter $L_{\text {eff }}$, and lower $D_{\text {eff. }}$ The closer the device is biased towards $J_{\text {sc }}$, the lower the effect of recombination is and the more the injection/regeneration processes dominate. This is in perfect agreement with previous works. ${ }^{16}$

\section{Optimization of p-type CuO DSSCs}

Optimization of the electrode calcination. In the preparation of electrodes, sintering is one of the most crucial steps. For instance, the sintering temperature $\left(T_{\text {sint }}\right)$ governs crystallinity, conductivity, and surface area. We doctorbladed a paste consisting of commercial CuO particles and ethyl cellulose diluted in ethanol on fluoride doped tin oxide (FTO) slides - for details see Experimental section - and investigated different $T_{\text {sint }}$, namely $300,350,400$, and $500{ }^{\circ} \mathrm{C}$.

The crystallinity of the sintered $\mathrm{CuO}$ electrodes was confirmed by X-ray diffraction (XRD) assays (Fig. S5 $\dagger$ ). ${ }^{17}$ Upon increasing $T_{\text {sint }}$, a sharpening of the diffraction peaks was noted. According to the Scherrer equation, the particle sizes were determined to be $14.0,15.0,19.0$, and $26.4 \mathrm{~nm}$ for 300 , 350, 400, and $500{ }^{\circ} \mathrm{C}$, respectively. ${ }^{33}$ Additional Raman assays show the characteristic CuO Raman modes, that is, $A_{g}$ at $275 \mathrm{~cm}^{-1}, \mathrm{~B}_{1 \mathrm{~g}}$ at $323 \mathrm{~cm}^{-1}$, and $\mathrm{B}_{2 \mathrm{~g}}$ at $610 \mathrm{~cm}^{-1}-\mathrm{Fig} . \mathrm{S} 6 . \dagger^{34}$ Additionally, the positions of $E_{\mathrm{F}}$ and the band gaps of the electrodes were determined by Kelvin Probe microscopy and diffuse reflectance measurements, respectively. ${ }^{35}$ The band gap energy $\left(\mathrm{E}_{\mathrm{g}}\right)$ is an all cases around $1.6 \mathrm{eV}-$ Fig. S7 $\dagger-$ while the position of $E_{\mathrm{F}}$ varied from 0.52 to $0.59 \mathrm{~V} v s$. NHE.

To gather insights into the film morphology and the particle structures, scanning electron microscopy (SEM) measurements were performed on the calcinated electrodes - Fig. S8. $\dagger$

Using $T_{\text {sint }}$ of 300 to $350{ }^{\circ} \mathrm{C}$, the $\mathrm{CuO}$ particles reveal rodlike structures of similar sizes and shapes. However, $T_{\text {sint }}$ from 400 to $500{ }^{\circ} \mathrm{C}$ result in films with round $\mathrm{CuO}$ particles and a higher overall particle size. The length of the rod-like CuO particles did not exceed $100 \mathrm{~nm}$ with a width from about 10 to $50 \mathrm{~nm}$, when sintered at $T_{\text {sint }} \leq 350{ }^{\circ} \mathrm{C}$, while the diameter of the spherical particles increases to $100 \mathrm{~nm}$ at $T_{\text {sint }} \geq 400$. Overall, SEM images prompt to a decrease of film porosity and particle surface upon increasing $T_{\text {sint }}$. Considering that BET measurements on the calcinated films afforded meaningless values we turned to dye desorption assays, which provide accurate estimations of the film roughness. ${ }^{36}$ The latter corroborate our hypothesis (Table 1) - vide infra.

To test the impact of the calcination temperature on the device performance, we built cells with all of the aforementioned $\mathrm{CuO}$ electrodes - see Experimental section for more details. The resulting $J-V$ curves and IPCE spectra are depicted in Fig. 4, while the corresponding EIS parameters and the figures-of-merit are summarized in Tables 1 and $\mathrm{S} 3, \uparrow$ respectively.

Devices calcinated at $300{ }^{\circ} \mathrm{C}$ showed the highest $J_{\text {sc }}$ with $2.76 \mathrm{~mA} \mathrm{~cm}^{-2}$, while higher $T_{\text {sint }}$ led to a decrease in $J_{\mathrm{sc}}$ to 2.53 $\left(350{ }^{\circ} \mathrm{C}\right), 1.79\left(400^{\circ} \mathrm{C}\right)$, and to $1.26 \mathrm{~mA} \mathrm{~cm}^{-2}\left(500^{\circ} \mathrm{C}\right)$. In corro- 
Table 1 Figures-of-merit and EIS parameters of p-type CuO DSSCs for the optimization of $T_{\text {Sint }}$, the electrode thickness and the electrolyte ratio

\begin{tabular}{|c|c|c|c|c|c|c|c|c|}
\hline \multicolumn{3}{|l|}{ Devices } & \multicolumn{2}{|c|}{ Device parameters } & \multicolumn{4}{|c|}{ Impedance parameters $^{a}$} \\
\hline $\begin{array}{l}\text { Sintering } \\
{\left[{ }^{\circ} \mathrm{C}\right]}\end{array}$ & $\begin{array}{c}\text { Thickness } \\
{[\mu \mathrm{m}]}\end{array}$ & $\mathrm{I}^{-} / \mathrm{I}_{2}$ ratio & $\begin{array}{c}\eta^{b} \\
{[\%]}\end{array}$ & $\begin{array}{l}\text { Dye loading }{ }^{c} \\
{\left[\mathrm{~mol} \mathrm{~cm}^{-2}\right]}\end{array}$ & $\begin{array}{c}R_{\mathrm{CT}} \\
{\left[\Omega \mathrm{cm}^{2}\right]}\end{array}$ & $\begin{array}{c}R_{\mathrm{rec}} \\
{\left[\Omega \mathrm{cm}^{2}\right]}\end{array}$ & $\begin{array}{l}\eta_{\mathrm{cc}} \\
{[\%]}\end{array}$ & $\begin{array}{c}\left.D_{\mathrm{eff}^{d}}{ }^{-1} \mathrm{~cm}^{2} \mathrm{~s}^{-1}\right]\end{array}$ \\
\hline \multicolumn{9}{|c|}{ Calcination temperature } \\
\hline 300 & 5.0 & $5: 1$ & 0.092 & 4.3 & $\begin{array}{r}8.3 \\
44.1\end{array}$ & $\begin{array}{l}20.9 \\
3.9 \mathrm{k}\end{array}$ & $\begin{array}{l}60.2 \\
98.8\end{array}$ & $\begin{array}{r}6.3 \\
49.0\end{array}$ \\
\hline 350 & 4.7 & $5: 1$ & 0.086 & 3.8 & $\begin{array}{r}9.3 \\
55.0\end{array}$ & $\begin{array}{l}23.7 \\
4.2 \mathrm{k}\end{array}$ & $\begin{array}{l}60.7 \\
98.6\end{array}$ & $\begin{array}{r}7.0 \\
44.0\end{array}$ \\
\hline 400 & 4.9 & $5: 1$ & 0.062 & 3.0 & $\begin{array}{l}14.2 \\
76.0\end{array}$ & $\begin{array}{l}32.7 \\
5.7 \mathrm{k}\end{array}$ & $\begin{array}{l}56.5 \\
98.6\end{array}$ & $\begin{array}{r}7.0 \\
44.0\end{array}$ \\
\hline 500 & 4.8 & $5: 1$ & 0.048 & 1.3 & $\begin{array}{r}15.2 \\
137.1\end{array}$ & $\begin{array}{l}36.9 \\
7.3 \mathrm{k}\end{array}$ & $\begin{array}{l}58.8 \\
98.0\end{array}$ & $\begin{array}{l}16.0 \\
41.0\end{array}$ \\
\hline \multicolumn{9}{|c|}{ Electrode thickness } \\
\hline 300 & 1.5 & $5: 1$ & 0.087 & 0.9 & $\begin{array}{l}14.9 \\
90.4\end{array}$ & $\begin{array}{l}38.2 \\
11.9 \mathrm{k}\end{array}$ & $\begin{array}{l}60.9 \\
99.2\end{array}$ & $\begin{array}{r}1.4 \\
16.0\end{array}$ \\
\hline 300 & 5.0 & $5: 1$ & 0.092 & 4.3 & $\begin{array}{r}8.3 \\
44.7\end{array}$ & $\begin{array}{l}20.9 \\
3.9 \mathrm{k}\end{array}$ & $\begin{array}{l}60.2 \\
98.8\end{array}$ & $\begin{array}{r}6.3 \\
49.0\end{array}$ \\
\hline 300 & 9.0 & $5: 1$ & 0.087 & 13.8 & $\begin{array}{r}6.8 \\
30.7\end{array}$ & $\begin{array}{l}15.7 \\
1.8 \mathrm{k}\end{array}$ & $\begin{array}{l}56.7 \\
98.0\end{array}$ & $\begin{array}{l}10.0 \\
68.4\end{array}$ \\
\hline \multicolumn{9}{|c|}{ Electrolyte ratio $\mathrm{I}^{-} / \mathbf{I}_{2}{ }^{-}$} \\
\hline 300 & 5.1 & $10: 1$ & 0.066 & 4.3 & $\begin{array}{l}13.5 \\
64.8\end{array}$ & $\begin{array}{l}31.1 \\
5.2 \mathrm{k}\end{array}$ & $\begin{array}{l}56.5 \\
98.7\end{array}$ & $\begin{array}{r}4.2 \\
39.0\end{array}$ \\
\hline 300 & 5.1 & $7.5: 1$ & 0.079 & 4.3 & $\begin{array}{l}11.9 \\
54.7\end{array}$ & $\begin{array}{l}24.4 \\
3.9 \mathrm{k}\end{array}$ & $\begin{array}{l}51.4 \\
98.6\end{array}$ & $\begin{array}{r}4.0 \\
39.0\end{array}$ \\
\hline 300 & 5.0 & $5: 1$ & 0.092 & 4.3 & $\begin{array}{r}8.3 \\
44.3\end{array}$ & $\begin{array}{l}20.9 \\
3.9 \mathrm{k}\end{array}$ & $\begin{array}{l}60.2 \\
98.8\end{array}$ & $\begin{array}{r}6.3 \\
49.0\end{array}$ \\
\hline 300 & 5.1 & $2.5: 1$ & 0.112 & 4.3 & $\begin{array}{r}5.8 \\
31.6\end{array}$ & $\begin{array}{l}14.0 \\
1.8 \mathrm{k}\end{array}$ & $\begin{array}{l}58.3 \\
97.7\end{array}$ & $\begin{array}{r}7.9 \\
29.0\end{array}$ \\
\hline
\end{tabular}

${ }^{a}$ All upper values were recorded at $V_{\text {oc }}$ and all lower values were recorded at $J_{\text {sc }}$ conditions. ${ }^{b}$ Device efficiency. ${ }^{c}$ All values multiplied by $10^{-9}$. ${ }^{d}$ All values multiplied by $10^{-5}$.
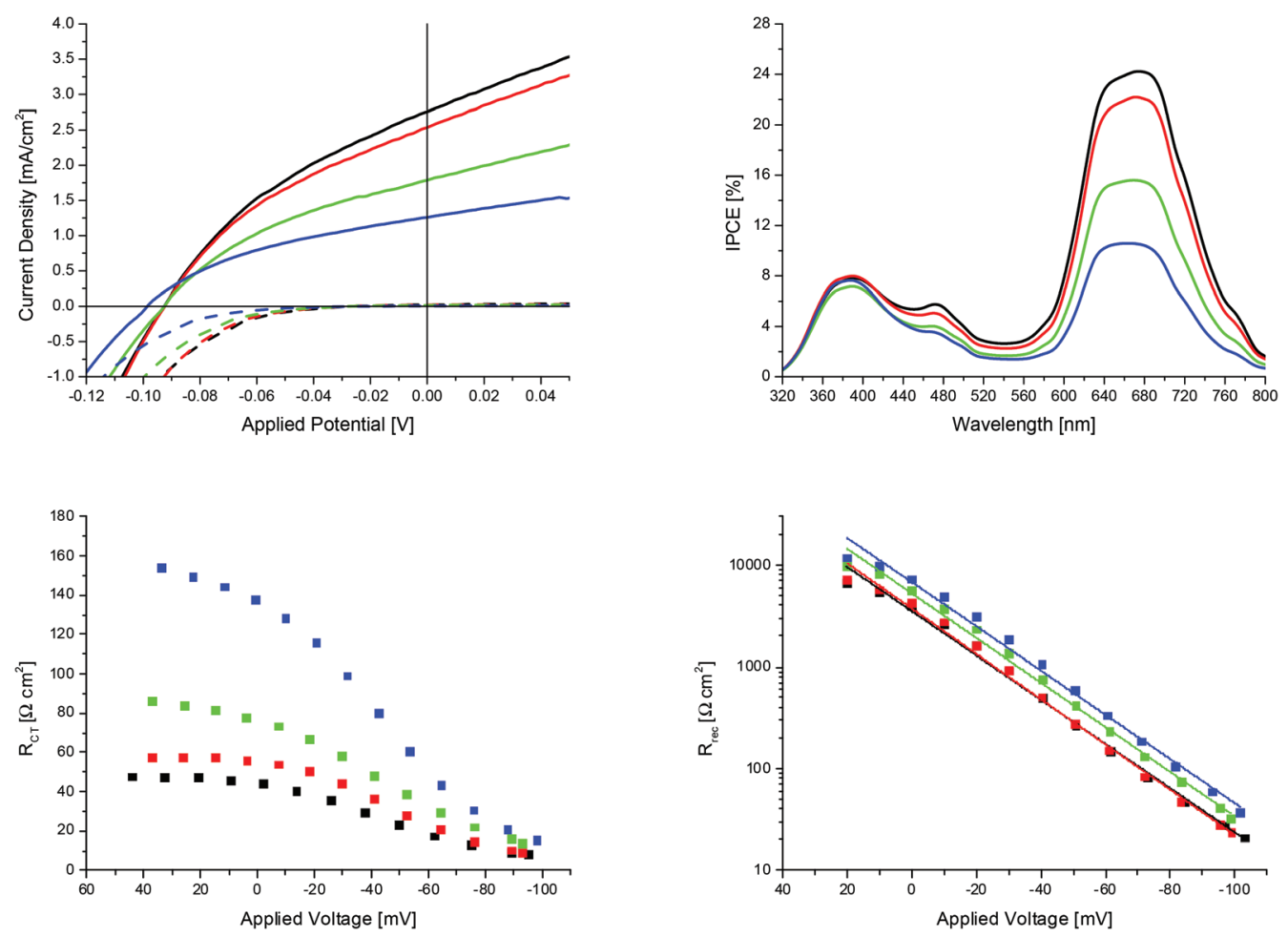

Fig. 4 Top left - Current density vs. applied potential under AM 1.5 (line) and dark (dashed) conditions. Top right - IPCE spectra. Bottom left - $R_{\mathrm{CT}}$ vs. applied voltage (light). Bottom right $-R_{\text {rec }} v s$. applied voltage (dark) with the measured resistances (points) and the corresponding fits (lines) according to eqn (8). The color code relates to devices with electrodes calcinated at 300 (black), 350 (red), 400 (green), and $500{ }^{\circ} \mathrm{C}$ (blue). 
boration of the $J_{\mathrm{sc}} \mathrm{S}$, devices calcinated at $300{ }^{\circ} \mathrm{C}$ show the highest IPCE at $670 \mathrm{~nm}$ with $24.3 \%$ compared to $22.3 \%$, $15.6 \%$, and $10.6 \%$ for increasing $T_{\text {sint }}$, respectively. $V_{\mathrm{oc}} \mathrm{s}$ were constant at around $92.6 \mathrm{mV}$, except for $500{ }^{\circ} \mathrm{C}$ calcinated devices, in which the $V_{\text {oc }}$ is $97.5 \mathrm{mV}$. Concerning FF, constant values of about 0.36 to 0.39 were noted. Taking all of the aforementioned into account, the most efficient devices were found at $T_{\text {sint }}$ of $300{ }^{\circ} \mathrm{C}$ with an overall efficiency of $0.092 \%$. An increase in temperature lowered the efficiencies from $0.086 \%$ to $0.062 \%$ and to $0.048 \%$ for 350,400 , and $500{ }^{\circ} \mathrm{C}$, respectively. Equal electrode thicknesses $(4.7-5.0 \mu \mathrm{m})$, comparable energetics, and only a slight increase in charge carrier mobility ( $\sim 20 \%$ ) upon increasing $T_{\text {sint }}{ }^{37}$ ensure that all changes in the device-figures-of merit are solely due to the change in calcination temperature, which rules the electrode morphology - vide supra.

XRD and SEM assays point to a change in particle size/morphology upon increasing $T_{\text {sint }}$ - vide supra. Here, dye desorption experiments corroborate a decrease of the amount of dye loading as a function of $T_{\text {sint }}$ - Table 1 .

Thus, devices prepared at $300{ }^{\circ} \mathrm{C}$ outperform the others due to the highest amount of dye loading $/ J_{\mathrm{sc}}$. It remains, however, unknown at this point, if sintering and the corresponding changes of film morphology could also affect mechanistic aspects. Thus, the impact of sintering on the charge injection and recombination processes in our devices was analyzed by means of EIS. In the dark, the measurements show an exponential increase in $R_{\text {rec }}$ with a decrease of voltage - Fig. 4 . Also, $R_{\text {rec }}$ increases as $T_{\text {sint }}$ is increased. This is reasonable, since an increase in $T_{\text {sint }}$ relates to lower surface areas of the $\mathrm{CuO}$ electrode. The latter leads to a suppression of recombination processes due to a lower probability of hole interception by the redox couple at the electrode/electrolyte interface.

The same trend is seen in EIS measurements at $V_{\text {oc }}$ under light - Fig. 4. Here, $R_{\mathrm{CT}}$ increases in the order $8.3<9.3<14.2<$ $15.2 \Omega \mathrm{cm}^{2}$ for temperatures of $300,350,400$, and $500{ }^{\circ} \mathrm{C}$, respectively. As already stated, $R_{\mathrm{CT}}$ increases before reaching a plateau at around $J_{\mathrm{sc}}$ following the order $44.1\left(300{ }^{\circ} \mathrm{C}\right), 55.0$ $\left(350{ }^{\circ} \mathrm{C}\right), 76.0\left(400{ }^{\circ} \mathrm{C}\right)$, and $137.1 \Omega \mathrm{cm}^{2}\left(500{ }^{\circ} \mathrm{C}\right)$. By relating the overall device performance to this finding, it is evident that a decrease in $R_{\mathrm{CT}}$ under $J_{\mathrm{sc}}$ conditions yields more efficient devices. Since the device performance is mainly influenced by the difference in dye loading - vide supra - it is reasonable to relate $R_{\mathrm{CT}}$ at $J_{\mathrm{sc}}$ and under light to the electron injection and regeneration. In this particular case, higher amount of dye loading leads to a higher net amount of charge injection and regeneration, which, subsequently, lowers the total value of $R_{\mathrm{CT}}$. A higher rate of charge injection/regeneration is also reflected in $C_{\mu}$ - Fig. $\mathrm{S} 9, \dagger$ which follows the trend of dye loading and $J_{\mathrm{sc}} \mathrm{s}$.

Concerning $\tau_{\text {dark }}$ and $\tau_{\text {light }}$, it is surprising that their trend is reverse to that of their corresponding resistances $R_{\text {rec }}$ and $R_{\mathrm{CT}}$, respectively - Fig. S9. $\dagger$ In detail, devices prepared at $300{ }^{\circ} \mathrm{C}$ show the longest lifetime under both light and dark, while an increase in $T_{\text {sint }}$ decreases $\tau_{\text {dark }}$ and $\tau_{\text {light }}$.
One has to consider the morphological changes and its influence on the trap states in the material upon heating - vide supra. In mesoporous n-type semiconductors, the amount of traps is being reduced in the bulk electrode upon increasing $T_{\text {sint }}$ as it becomes more crystalline. ${ }^{38,39}$ Electrodes calcinated at, for example, $300{ }^{\circ} \mathrm{C}$ show the highest $\tau_{\text {dark }}$ and $\tau_{\text {light }}$ due to the highest amount of traps. Furthermore, it has been shown that in $\mathrm{TiO}_{2}$-based DSSCs, recombination depends on the amount of trapped charge carriers. ${ }^{40}$ Applying this notion to our CuO-based DSSCs, it is evident that the trend of $R_{\mathrm{rec}}$ is in line with the number of trap states. In other words, more traps inside the electrode lead to a higher recombination rate/lower $R_{\text {rec }}$.

Another useful parameter, which shows the same trend as the device efficiency, is $L_{\text {eff }}-$ Fig. S9† and eqn (5). Although devices calcinated at $300{ }^{\circ} \mathrm{C}$ show the lowest $R_{\text {rec }}$ - the highest amount of recombination - they also possess the lowest $R_{\mathrm{CT}}$ at $J_{\mathrm{sc}}$ light conditions - highest amount of charge injection/ regeneration. Hence, devices prepared at $300{ }^{\circ} \mathrm{C}$ show a superior amount of injected charges that are filling up all the traps, allowing the free charge carriers to travel more easily. This compensates the higher recombination, which leads to $L_{\text {eff }} \mathrm{S}$ as large as 7.96 and $45.9 \mu \mathrm{m}$ at $V_{\mathrm{oc}}$ and $J_{\mathrm{sc}}$, respectively. By going to higher $T_{\text {sint }}$, the ratio between injection and recombination is shifted more towards the latter since the amount of injected charges drops significantly, which evolves in a lowering of $L_{\text {eff }}$.

More interesting is $D_{\text {eff }}$, which shows a reversed trend, when comparing $V_{\mathrm{oc}}$ and $J_{\mathrm{sc}}$ conditions - Fig. S9. $\dagger$ At $V_{\mathrm{oc}}$, the diffusion of VB holes is dictated by the rate of recombination, and therefore, devices calcinated at $500{ }^{\circ} \mathrm{C}$ with highest $R_{\text {rec}}$, feature the optimum hole diffusion. With decreasing voltages, this trend is reversed at roughly $-20 \mathrm{mV}$, at which point $300^{\circ} \mathrm{C}$ sintered devices showed the highest $D_{\text {eff }}$, while for the $500{ }^{\circ} \mathrm{C}$ sintered devices the poorest diffusion coefficients are seen. A likely rationale infers that at lower voltages the effect of recombination starts to diminish and the superior electron injection/ regeneration of the $300{ }^{\circ} \mathrm{C}$ calcinated devices takes over in terms of hole diffusion. In summary, despite sizeable recombination enough charge carriers are produced so that $D_{\text {eff }}$ is still at its highest for $300{ }^{\circ} \mathrm{C}$ sintered devices. $\eta_{\mathrm{cc}}$ is depicted in Fig. S9, $\dagger$ but shows only minor differences between the different $T_{\text {sint }}$.

Impact of the electrode thickness. CuO-DSSCs with thicknesses of 1.5, 5.0, and $9.0 \mu \mathrm{m}$ were prepared using $T_{\text {sint }}$ $300{ }^{\circ} \mathrm{C}$. The corresponding $J-V$ curves and IPCE spectra are shown in Fig. 5 and the EIS parameters and device figures-ofmerit are summarized in Tables 1 and S3, $\dagger$ respectively.

Upon increasing electrode thickness, $V_{\mathrm{oc}} \mathrm{s}$ decrease from 112.2 to 92.6 and to $82.9 \mathrm{mV}$, while $J_{\mathrm{sc}} \mathrm{s}$ increase from 2.01 to 2.76 and to $3.08 \mathrm{~mA} \mathrm{~cm}^{-2}$. Furthermore, the $J_{\mathrm{sc}}$ trend is corroborated by IPCEs at, for example, $670 \mathrm{~nm}$ with 17.7\%, 24.3\%, and $25.5 \%$ - Table S3. $\dagger$ At the same time, FFs decrease from 0.38 to 0.36 and to 0.34 . Interestingly, the device efficiencies scattered only between $0.087 \%$ and $0.092 \%$. Desorption experiments revealed the expected increase in dye loading and $J_{\mathrm{sc}} \mathrm{S}$ 

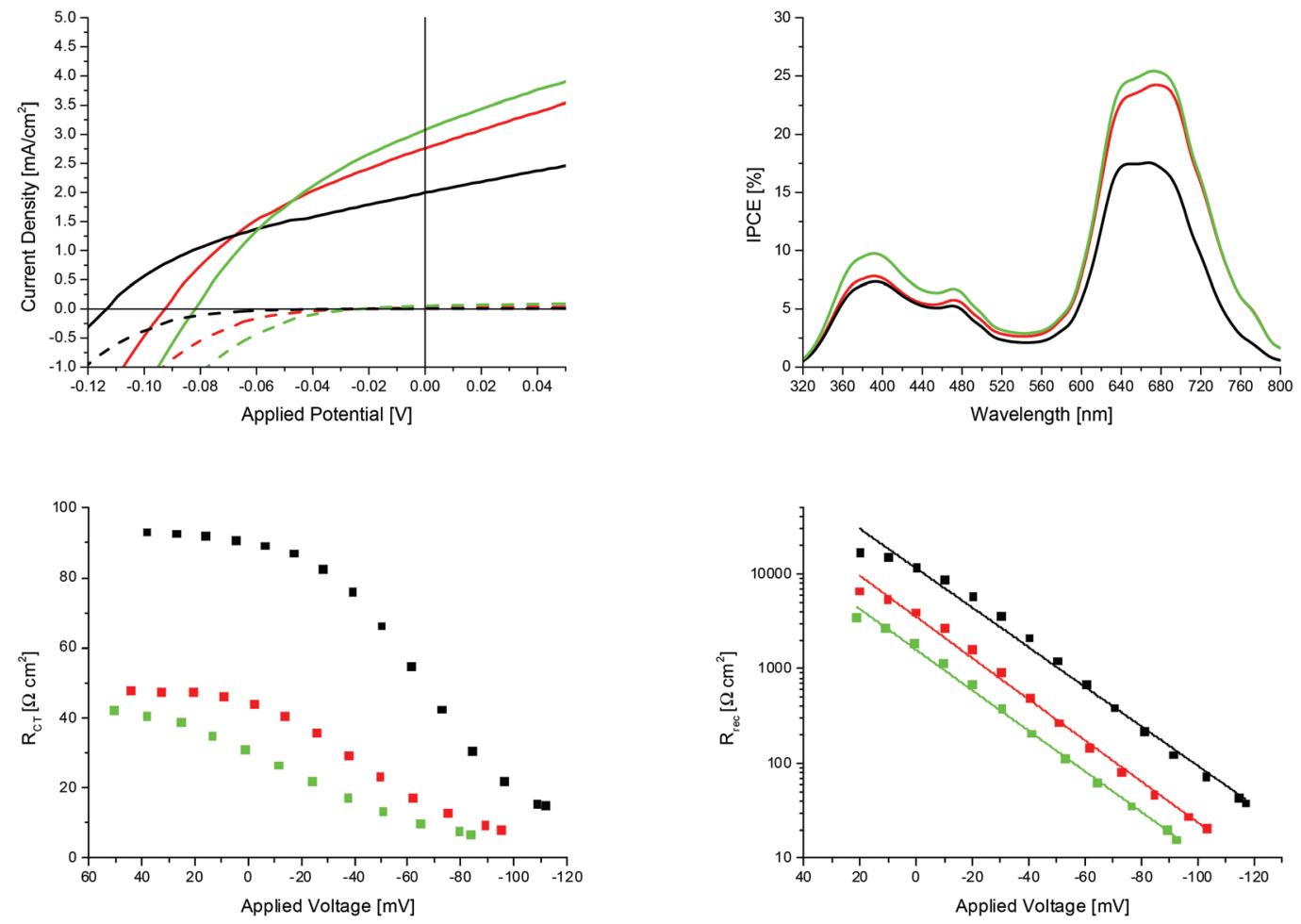

Fig. 5 Top left - Current density vs. applied potential under AM 1.5 (line) and dark (dashed) conditions. Top right - IPCE spectra. Bottom left $-R_{\mathrm{CT}}$ vs. applied voltage (light). Bottom right $-R_{\text {rec }} v s$. applied voltage (dark) with the measured resistances (points) and the corresponding fits (lines) according to eqn (8). The color code relates to devices with electrode thicknesses of 1.5 (black), 5.0 (red), and $9.0 \mu \mathrm{m}$ (green).

versus increase in film thickness - please compare $0.9 \times 10^{-9}$ $(1.5 \mu \mathrm{m})$ to $4.3 \times 10^{-9}(5.0 \mu \mathrm{m})$ and to $13.8 \times 10^{-9} \mathrm{~mol} \mathrm{~cm}^{-2}$ $(9.0 \mu \mathrm{m})$.

Thus, the differences in $J_{\mathrm{sc}} \mathrm{s}$ could be attributed to the different amount of dye loading, while the lowering of the film thickness might increase the shunt resistance, explaining the $\mathrm{FF}$ enhancement. Moreover, the differences in $V_{\mathrm{oc}} \mathrm{S}$ are explained by considering the balance between charge injection/transport and recombination processes. In thinner films, the redox couple has an overall shorter $L_{\text {eff }}$ through the mesoporous electrode, which leads to a lower recombination probability with the electrode and, thus, a high $V_{\text {oc }}$. In thicker films, recombination dominates and lower $V_{\mathrm{oc}} \mathrm{S}$ are typically noted. An electrode thickness of around $5.0 \mu \mathrm{m}$ seems to provide the best balance between $V_{\text {oc }}$ and $J_{\text {sc }}$, yielding a slightly higher efficiency than 1.5 and $9.0 \mu \mathrm{m}$ thick devices.

New insights are provided by EIS assays - Fig. 5. In the dark, $R_{\text {rec }}$ shows the expected exponential increase upon reducing the applied voltage. Devices with the thickest electrodes exhibited the lowest $R_{\text {rec}}$, while the thinnest electrodes show higher $R_{\text {rec}}$. Thus, we conclude that the recombination process must be enhanced and that the likelihood of the reduced electrolyte to recombine is influenced by the film thickness. Under light, $R_{\mathrm{CT}}$ shows the same trend. It increases at $V_{\mathrm{oc}}$ in the order of $6.8<8.3<14.9 \Omega \mathrm{cm}^{2}$ for thicknesses of 9.0, 5.0, and $1.5 \mu \mathrm{m}$, respectively, while at $J_{\mathrm{sc}}, R_{\mathrm{CT}}$ increases with a decrease in film thickness - Table 1 . Similar to the $T_{\text {sint }}$ assays, the trend at $J_{\mathrm{sc}}$ is governed by the dye loading. More efficient charge injection is also confirmed by the differences in $C_{\mu}$. Here, devices with thicker films show higher dye loadings, lower $R_{\mathrm{CT}} \mathrm{S}$, and higher capacitance - Fig. S10.† $\tau_{\text {dark }}$ and $\tau_{\text {light }}$ relate to $C_{\mu}-$ Fig. S10. $\dagger$ In the context of $\tau_{\text {dark }}$, nearly identical values have been derived for all three thicknesses, since low $R_{\text {rec }} \mathrm{S}$ are associated with high DOSs/high $C_{\mu} \mathrm{s}$ and vice versa. At $V_{\text {oc }}$ and under light, $\tau_{\text {light }}$ decreases in the order $10.3>7.6>$ $3.6 \mathrm{~ms}$ for thicknesses of 9.0, 5.0, and $1.5 \mu \mathrm{m}$, respectively, and the same trend can be seen at $J_{\text {sc }}$, where $\tau_{\text {light }}$ increases as we are going to thicker film.

This is explained by a higher probability of trapping in the case of thicker electrodes. Here, the chance for trapping increases at longer travel distances inside the electrode material. In addition, an increase in trapping $/ \tau_{\text {light }}$ leads to a higher rate of recombination - vide supra - which is again confirmed by the trend of $R_{\text {rec }}$.

Owing to the different thicknesses, we analyzed the $L_{\text {eff }} / d$ ratio, which could be interpreted as how many charges are ready to diffuse before recombining with the electrolyte Fig. S10. $\dagger$ Comparing with $R_{\text {rec }}$, a lower amount of recombination with the electrolyte correlates with a higher $L_{\mathrm{eff}} / d$ ratio. Finally, $\eta_{\text {cc }}$ increases at $V_{\text {oc }}$ in the order of $56.7 \%<60.2 \%<$ $60.9 \%$ for thicknesses 9.0, 5.0, and $1.5 \mu \mathrm{m}$, respectively Fig. S10. $\dagger$ Notably, for films with intermediate thicknesses, the charge collection is with $0.7 \%$ only slightly lower than for the $1.5 \mu \mathrm{m}$ devices, a fact that is offset by the total amount of 
collected charges at the back contact. In short, 5.0 $\mu \mathrm{m}$ devices show the best ratio between injection and recombination, which is reflected in the highest efficiency of $0.092 \%$.

Finding the right electrolyte composition. In the final step, we address the electrolyte composition. As mentioned in the introduction, please notice that in our previous study cobaltbased electrolytes revealed higher $V_{\mathrm{oc}} \mathrm{S}$ and, thus, higher efficiencies compared to iodine-based electrolytes. ${ }^{8}$ However, due to the fast crystallisation onto the electrode surface a low device stability was noted, and a complete EIS analysis of devices based on cobalt-based electrolytes could not be performed. Thus, in the following, we exclusively focus on devices filled with the iodine-based redox couple and especially the $\mathrm{I}^{-} /$ $\mathrm{I}_{2}$ ratios, which influence both the dye regeneration and recombination. To this end, we kept the LiI concentration constant at $1 \mathrm{M}$, while the $\mathrm{I}_{2}$ concentration was varied from 0.1 , $0.133,0.2$, to $0.4 \mathrm{M}$, resulting in acetonitrile-based electrolytes with $\mathrm{I}^{-} / \mathrm{I}_{2}$ molar ratios of $10: 1,7.5: 1,5: 1$, and $2.5: 1$, respectively. The $J-V$ curves and IPCE spectra are gathered in Fig. 6 and the EIS parameters and device figures-of-merit are summarized in Tables 1 and $\mathrm{S} 3, \uparrow$ respectively.

Highest $J_{\mathrm{sc}}$ s were found to be $3.57 \mathrm{~mA} \mathrm{~cm}^{-2}$ for the $2.5: 1$ ratio, while a lower $\mathrm{I}_{2}$ concentration in the electrolyte affects $J_{\mathrm{sc}}$ in terms of an overall decrease to $2.76,2.27$, and $1.92 \mathrm{~mA} \mathrm{~cm}^{-2}$ for molar ratios of $5: 1,7.5: 1$, and $10: 1$, respectively. The trend in $V_{\text {oc }}$ is opposite, namely it increases from 87.7 to 92.6 to 99.9 and to $95.1 \mathrm{mV}$ upon decreasing the $\mathrm{I}_{2}$ concentration in the electrolyte. No major changes were noted for the FFs. Different $\mathrm{I}^{-} / \mathrm{I}_{2}$ ratios induce a massive impact on the device efficiency, with the $2.5: 1$ ratio showing the highest efficiency of $0.112 \%$ followed by $0.092 \%(5: 1), 0.079 \%(7.5: 1)$, and $0.066 \%(10: 1)$. Not unexpected is the change in IPCE, which corroborates the $J_{\mathrm{sc}}$ trend, decreasing from $29.6 \%$ to $17.1 \%$ for the 2.5:1 and 10:1 molar ratios, respectively. In p-type NiO DSSCs, an increase in the $\mathrm{I}_{2}$ concentration of the electrolyte raises the photocurrents, which is related to the excitation of the redox couple due to its absorption in the visible light spectrum. ${ }^{41,42}$ In CuO-DSSCs contributions stemming from the excitation of $\mathbf{Z n P c 2}$ sensitizer increased rather than plateauing as the $I_{2}$ concentration in the electrolyte reached a critical cutoff. Even at very high iodine concentrations - 2.5:1 ratio it still is the major pathway of the photocurrent generation. Since the $\mathrm{I}^{-} / \mathrm{I}_{2}$ ratio of the redox couple is the only variable here, we conclude that a higher amount of iodine favors the regeneration of the excited dye by the electrolyte and, in turn, yields a higher $J_{\mathrm{sc}}$. This trend is unique and has never been observed in p-type DSSCs.

Next, EIS assays were performed - Fig. 6. By increasing the iodine concentration in the electrolyte two parameters are impacted. On one hand, the redox potential of the electrolyte shifts to more positive values. An immediate consequence is a lower theoretical $V_{\mathrm{oc}}$ for p-type DSSCs, as well as a slightly higher driving force for the dye regeneration. We calculated a shift on the order of $30 \mathrm{mV}$ when comparing the 10:1 and
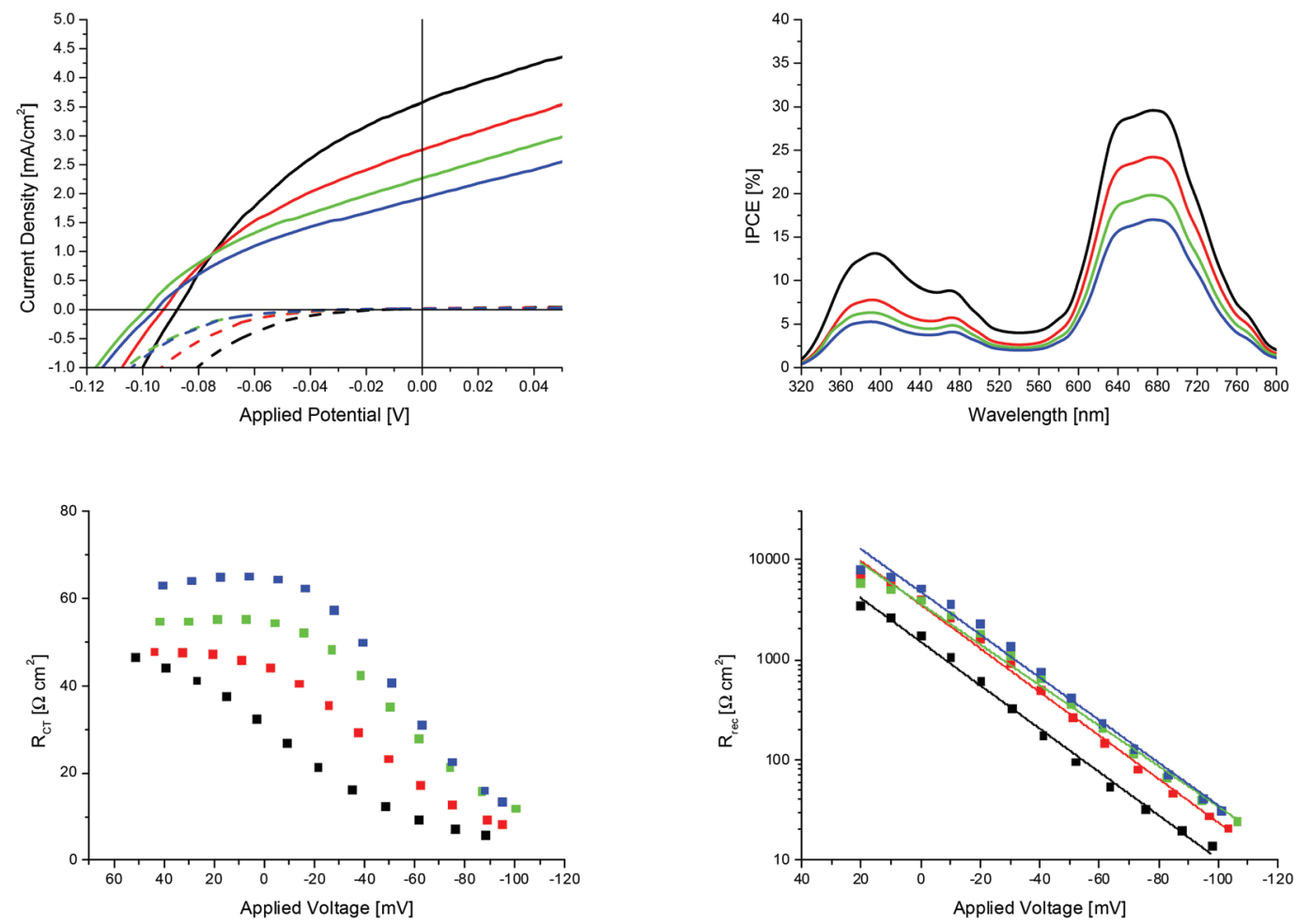

Fig. 6 Top left - Current density vs. applied potential under AM 1.5 (line) and dark (dashed) conditions. Top right - IPCE spectra. Bottom left $-R_{C T}$ vs. applied voltage (light). Bottom right $-R_{\text {rec }} v s$. applied voltage (dark) with the measured resistances (points) and the corresponding fits (lines) according to eqn (8). The color code relates to devices with electrolyte ratios of $2.5: 1$ (black), $5: 1$ (red), $7.5: 1$ (green), and $10: 1$ (blue). 
2.5: 1 ratios - please see the $\mathrm{ESI} \dagger$ for a detailed explanation of the calculations. ${ }^{43}$ A correlation with our device figures-ofmerit (Table S3†) prompts indeed to lower $V_{\mathrm{oc}} \mathrm{S}$ for an increase in iodine concentrations - vide supra. On the other hand, an increased iodine concentration results in a higher amount of active redox species in the electrolyte. This assists in the regeneration of the excited photosensitizer, but also a higher amount of recombination is expected. A comparison of the $R_{\mathrm{CT}} \mathrm{S}$ at $V_{\mathrm{oc}}$ and under illumination shows a clear trend for the electrolyte compositions.

Lower iodine concentrations are linked to higher $R_{\mathrm{CT}} \mathrm{S}$ with values of $5.8,8.3,11.9$, and $13.5 \Omega \mathrm{cm}^{2}$ for molar ratios of $2.5: 1,5: 1,7.5: 1$, and $10: 1$, respectively. The same trend is derived for the EIS measurements of $R_{\text {rec }}$ in the dark. Here, an increase in the amount of iodine lowers $R_{\text {rec }}$ over the whole voltage range, which points a higher recombination with the electrode surface. This substantiates the notion that a higher amount of iodine in the electrolyte produces more active species, which can undergo a higher total amount of recombination with the electrode surface. It is, however, counterintuitive to think that devices with maximized recombination, would give rise to the highest efficiency. Helpful is in this regard to inspect $R_{\mathrm{CT}}$ at $J_{\mathrm{sc}}$ and under light. The electron injection is equal for all the devices, since all the electrode parameters were constant and the same sensitizer was used. The only process that has not been considered so far is the dye regeneration by means of the electrolyte. Taking the $J_{\mathrm{sc}}$ contribution from the sensitizer - IPCE maximum at $670 \mathrm{~nm}$ - into account, a clear trend between $R_{\mathrm{CT}} \mathrm{S}$ and the dye regeneration is discernible. Here, we note that the $2.5: 1$ molar ratio has the lowest $R_{\mathrm{CT}}$ with $31.6 \Omega \mathrm{cm}^{2}$ and at the same time the highest $J_{\mathrm{sc}}$ and IPCE at $670 \mathrm{~nm}$ with $3.57 \mathrm{~mA} \mathrm{~cm}^{-2}$ and $29.6 \%$, respectively. A lowering of the iodine concentration affects $R_{\mathrm{CT}}$ with values of $44.3,54.7$, and $64.8 \Omega \mathrm{cm}^{2}$ for the $5: 1,7.5: 1$, and $10: 1$ molar ratios, respectively, which goes in line with a decreasing $J_{\mathrm{sc}}$ and IPCE at $670 \mathrm{~nm}$ - please see the device figures-of-merit in Table S3. $\dagger$ This points to a superior regeneration for the $2.5: 1$ molar ratio.

Next, the $C_{\mu}$ s provide valuable insights into the amount of charges, which are produced in each individual step. Fig. S11 $\dagger$ shows that an increase in $\mathrm{I}_{2}$ and, thus, also the $\mathrm{I}_{3}{ }^{-}$concentration go hand-in-hand with an increase of $C_{\mu}$ across the whole voltage region. This indicates the presence of more charge carriers, leading to a higher $J_{\mathrm{sc}}$.

Considering $\tau_{\text {light }}$ and $\tau_{\text {dark }}$ minor differences compared to the $T_{\text {sint }}$ and electrode thickness measurements are noticeable. Fig. S11† reveals that the trend for $\tau_{\text {light }}$ is dominated by $R_{\mathrm{CT}}$, owing to the fact that differences in $C_{\mu}$ are smaller than in the above mentioned experiments. The major difference is here that the recombination rate of the trapped charges is only dependent on the electrolyte properties. Therefore, $\tau_{\text {light }}$ increases as the iodine concentration is decreased in the electrolyte. At $V_{\text {oc }}$, low amounts of iodine seemingly connect with a lower rate of recombination and, thus, a longer lifetime. At $J_{\mathrm{sc}}$, $\tau_{\text {light }}$ reflects the regeneration. As expected, a lower $R_{\mathrm{CT}}$ yields a lower time constant - 24.5 (2.5:1), 35.4 (5:1), 40.9 (7.5:1), and $45.1 \mathrm{~ms}(10: 1)$ and therefore a more efficient regeneration process. In the dark, $R_{\text {rec }}$ also dictates $\tau_{\text {dark }}$ with the shortest lifetime for the 2.5:1 ratio and longer lifetimes at lower iodine concentrations - Fig. S11. $\dagger$

Finally, we calculated $\eta_{\mathrm{cc}}, L_{\mathrm{eff}}, D_{\mathrm{eff}}$ under light and in the dark - Fig. S11. $\dagger$ Owing due to relatively small $R_{\mathrm{rec}} \mathrm{S}$, device with 2.5:1 electrolytes possess the lowest $\eta_{\mathrm{cc}}$. Still enough charges are produced to reach the highest efficiency and $J_{\mathrm{sc}}$. A 5:1 ratio shows the best balance between regeneration and recombination processes, as the highest $\eta_{\mathrm{cc}}$ indicates. As the effective diffusion length $L_{\text {eff }}$ relates to $\eta_{\mathrm{cc}}$, for the different ratios the same trend, that is, the 2.5:1 and 5:1 molar ratios, showing the shortest and the longest $L_{\text {eff }}$, respectively. $D_{\text {eff }}$ shows trends differing at $V_{\mathrm{oc}}$ and $J_{\mathrm{sc}}$. At $V_{\mathrm{oc}}, C_{\mu}$ has a major influence on $D_{\text {eff }}$, since here the largest differences evolve and, thus, a 2.5: 1 molar ratio features both the highest $C_{\mu}$ and $D_{\text {eff. }}$ By lowering the voltage, the differences in $C_{\mu}$ become smaller and the $R_{\text {rec }} / R_{\mathrm{CT}}$ ratio is in control over the diffusion coefficients. Here, using a 5:1 electrolyte ratio leads to the most favorable ratio of recombination and regeneration/injection,

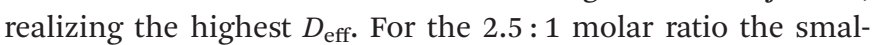
lest $D_{\text {eff }}$ are noted. Even though the $5: 1$ molar ratio possesses the best performing $\eta_{\mathrm{cc}}, L_{\mathrm{eff}}$, and $D_{\text {eff }}$, the $2.5: 1$ molar ratio still has the most efficient dye regeneration process, due to the lowest $R_{\mathrm{CT}}$, which, in turn, is reflected in the highest IPCE and efficiency.

\section{Conclusions}

A new and comprehensive EIS interpretation, which enables us to effectively separate the recombination from the electron injection and regeneration processes, is proposed. With the help of this approach, we studied the effect of different device parameters on $R_{\mathrm{rec}} \mathrm{S}$ and $R_{\mathrm{CT}} \mathrm{S}$ in concert with advanced EIS parameters like $\eta_{\mathrm{cc}}, D_{\text {eff }}, L_{\text {eff }}, \tau_{\text {light }}$, and $\tau_{\text {dark }}$ as a function of calcination temperature, electrode thickness, and electrolyte ratio. In short, a calcination temperature of $300^{\circ} \mathrm{C}$ and a film thickness of $5.0 \mu \mathrm{m}$ yielded the best balance between dye loading, charge injection, and recombination, with an $\mathrm{I}^{-} / \mathrm{I}_{2}{ }^{-}$ electrolyte ratio of $2.5: 1$ as the best choice for the dye regeneration. To this end, these optimized devices based on the iodide/triiodide redox couple feature efficiencies of up to $0.11 \%$, which is 10 -fold higher than the state-of-the-art $\mathrm{CuO}$ devices and comparable to the efficiencies recently obtained by our group. ${ }^{3,8}$ Future work will focus on the stability and EIS characterization of cobalt-based electrolytes, in order to underline the reasons of their superior performance compared to the iodide/triiodide redox couple.

In addition, the effect of trap states on the $\tau_{\text {light }}$ and $\tau_{\text {dark }}$ has been studied for the first time in p-type DSSCs by EIS. For the above mentioned variables different observations have been obtained. In the context of $T_{\text {sint }}$ and the electrode thickness, big differences in $C_{\mu}$ were governing $\tau_{\text {light }}$ and $\tau_{\text {dark }}$, which lead to longer lifetimes and increased recombination for a higher amount of trapping. For the different electrolyte 
molar ratios, the differences in $C_{\mu}$ were small and, thus, $R_{\mathrm{CT}}$ and $R_{\text {rec }}$ showed a major impact on $\tau_{\text {light }}$ and $\tau_{\text {dark }}$. Here, a shorter lifetime pointed to a faster recombination of the trapped charge carriers.

Furthermore, the reactivity of non-sensitized $\mathrm{CuO}$ electrodes with the electrolyte solution was probed by EIS measurements in the dark and compared to state-of-the-art NiO electrodes. Surprisingly, $\mathrm{CuO}$ electrodes showed a lower reactivity towards the redox couple compared to NiO electrodes.

\section{Acknowledgements}

The authors thank the German Science Council (DFG) for the financial support in the framework of the Cluster of Engineering of Advanced Materials (EAM), the MINECO Spain (CTQ2014-52869/BQU), the Comunidad de Madrid Spain (FOTOCARBON, S2013/MIT-2841), and the European Union within the FP7-ENERGY-2012-1 nr. 309194-2, GLOBALSOL.

\section{Notes and references}

$1 \mathrm{~J} . \mathrm{He}, \mathrm{H}$. Lindström, A. Hagfeldt and L. Sten-Eric, J. Phys. Chem. B, 1999, 103, 8940-8943.

2 F. Odobel and Y. Pellegrin, J. Phys. Chem. Lett., 2013, 4, 2551-2564.

3 S. Sumikura, S. Mori, S. Shimizu, H. Usami and E. Suzuki, J. Photochem. Photobiol., A, 2008, 194, 143-147.

4 D. Xiong, Z. Xu, X. Zeng, W. Zhang, W. Chen, X. Xu, M. Wang and Y.-B. Cheng, J. Mater. Chem., 2012, 22, 24760-24768.

5 M. Yu, G. Natu, Z. Ji and Y. Wu, J. Phys. Chem. Lett., 2012, 3, 1074-1078.

6 A. Nattestad, X. Zhang, U. Bach and Y.-B. Cheng, J. Photonics Energy, 2011, 1, 1-9.

7 M. Yu, T. I. Draskovic and Y. Wu, Phys. Chem. Chem. Phys., 2014, 16, 5026-5033.

8 O. Langmar, C. R. Ganivet, A. Lennert, R. D. Costa, G. de la Torre, T. Torres and D. M. Guldi, Angew. Chem., Int. Ed., 2015, 54, 7688-7692.

9 J. Bisquert, J. Phys. Chem. B, 2002, 106, 325-333.

10 F. Fabregat-Santiago, G. Garcia-Belmonte, I. Mora-Seró and J. Bisquert, Phys. Chem. Chem. Phys., 2011, 13, 9083-9118.

11 J. Yang, P. Ganesan, J. Teuscher and M. Grätzel, J. Am. Chem. Soc., 2014, 136, 5722-5730.

12 C. Magne, T. Moehl, M. Urien, M. Grätzel and T. Pauporté, J. Mater. Chem. A, 2013, 1, 2079-2088.

13 J. Bisquert, F. Fabregat-Santiago, I. Mora-Seró, G. GarciaBelmonte and S. Giménez, J. Phys. Chem. C, 2009, 113, 17278-17290.

14 J. Bisquert, Phys. Chem. Chem. Phys., 2003, 5, 5360-5364.

15 Z. Huang, G. Natu, Z. Ji, P. Hasin and Y. Wu, J. Phys. Chem. $C, 2011,115,25109-25114$.

16 Z. Huang, G. Natu, Z. Ji, M. He, M. Yu and Y. Wu, J. Phys. Chem. C, 2012, 116, 26239-26246.
17 D. M. Jundale, P. B. Joshi, S. Sen and V. B. Patil, J. Mater. Sci.: Mater. Electron., 2012, 23, 1492-1499.

18 S. Makhlouf, M. Kassem and M. Abdel-Rahim, J. Mater. Sci., 2009, 44, 3438-3444.

19 B. Boukamp, J. Electrochem. Soc., 1995, 142, 1885-1894.

20 Y. Y. Proskuryakov, K. Durose, M. K. Al Turkestani, I. MoraSeró, G. Garcia-Belmonte, F. Fabregat-Santiago, J. Bisquert, V. Barrioz, D. Lamb, S. J. C. Irvine and E. W. Jones, J. Appl. Phys., 2009, 106, 044507.

21 J. Idígoras, G. Burdziński, J. Karolczak, J. Kubicki, G. Oskam, J. a. Anta and M. Ziółek, J. Phys. Chem. C, 2015, 119, 3931-3944.

22 T. Daeneke, Z. Yu, G. P. Lee, D. Fu, N. W. Duffy, S. Makuta, Y. Tachibana, L. Spiccia, A. Mishra, P. Bäuerle and U. Bach, Adv. Energy Mater., 2015, 5, 1-11.

23 Q. Wang, S. Ito, M. Grätzel, F. Fabregat-Santiago, I. MoraSeró, J. Bisquert, T. Bessho and H. Imai, J. Phys. Chem. B, 2006, 110, 25210-25221.

24 Q. Wang, J.-E. Moser and M. Grätzel, J. Phys. Chem. B, 2005, 109, 14945-14953.

25 M. Liberatore, F. Decker, L. Burtone, V. Zardetto, T. M. Brown, A. Reale and A. Di Carlo, J. Appl. Electrochem., 2009, 39, 2291-2295.

26 M. Adachi, M. Sakamoto and J. Jiu, J. Phys. Chem. B, 2006, 110, 13872-13880.

27 J. Song, Z. Yin, Z. Yang, P. Amaladass, S. Wu, J. Ye, Y. Zhao, W.-Q. Deng, H. Zhang and X.-W. Liu, Chem. - Eur. J., 2011, 17, 10832-10837.

28 F. Fabregat-Santiago, G. Garcia-Belmonte, J. Bisquert, A. Zaban and P. Salvador, J. Phys. Chem. B, 2002, 106, 334339.

29 L. D. Amario, G. Boschloo, A. Hagfeldt and L. Hammarström, J. Phys. Chem. C, 2014, 118, 1955619564.

30 S. Sumikura, S. Mori, S. Shimizu, H. Usami and E. Suzuki, J. Photochem. Photobiol., A, 2008, 199, 1-7.

31 E. Guillén, L. M. Peter and J. A. Anta, J. Phys. Chem. C, 2011, 115, 22622-22632.

32 M. Wang, P. Chen, R. Humphry-Baker, S. M. Zakeeruddin and M. Grätzel, ChemPhysChem, 2009, 10, 290-299.

33 A. L. Patterson, Phys. Rev., 1939, 56, 978-982.

34 A. Bhaumik, A. M. Shearin, R. Patel and K. Ghosh, Phys. Chem. Chem. Phys., 2014, 16, 11054-11066.

35 V. Kumar, S. K. Sharma, T. Sharma and V. Singh, Opt. Mater., 1999, 12, 115-119.

36 R. D. Costa, S. Feihl, A. Kahnt, S. Gambhir, D. L. Officer, G. G. Wallace, M. I. Lucio, M. A. Herrero, E. Vázquez, Z. Syrgiannis, M. Prato and D. M. Guldi, Adv. Mater., 2013, 25, 6513-6518.

37 D. M. Jundale, P. B. Joshi, S. Sen and V. B. Patil, J. Mater. Sci.: Mater. Electron., 2012, 23, 1492-1499.

38 K. Takeshita, Y. Sasaki, M. Kobashi, Y. Tanaka, S. Maeda, A. Yamakata, T. Ishibashi and H. Onishi, J. Phys. Chem. B, 2004, 108, 2963-2969.

39 D. Zhao, T. Peng, L. Lu, P. Cai, P. Jiang and Z. Bian, J. Phys. Chem. C, 2008, 112, 8486-8494. 
40 J. Nelson, S. Haque, D. Klug and J. Durrant, Phys. Rev. B: Condens. Matter, 2001, 63, 1-9.

41 J. Cong, X. Yang, L. Kloo and L. Sun, Energy Environ. Sci., 2012, 5, 9180-9194.

42 Z. Hongjun, A. Hagfeldt and G. Boschloo, J. Phys. Chem. C, 2007, 111, 17455-17458.
43 G. Boschloo and A. Hagfeldt, Acc. Chem. Res., 2009, 42, 1819-1826.

44 S. Feihl, R. D. Costa, W. Brenner, J. T. Margraf, R. Casillas, O. Langmar, A. Browa, T. E. Shubina, T. Clark, N. Jux and D. Guldi, Chem. Commun., 2014, 50, 11339-11342. 